\title{
Nonlinear Robust Disturbance Attenuation Control Design for Static Var Compensator in Power System
}

\author{
Ting Liu, Nan Jiang, Yuanwei Jing, and Siying Zhang \\ Faculty of Information Science and Engineering, Northeastern University, Box 135 Shenyang, Liaoning 110819, China \\ Correspondence should be addressed to Ting Liu; liuting_tinka@sina.cn
}

Received 23 August 2013; Accepted 28 October 2013

Academic Editor: Baoyong Zhang

Copyright (C) 2013 Ting Liu et al. This is an open access article distributed under the Creative Commons Attribution License, which permits unrestricted use, distribution, and reproduction in any medium, provided the original work is properly cited.

\begin{abstract}
The problem of designing an adaptive backstepping controller for nonlinear static var compensator (SVC) system is addressed adopting two perspectives. First, instead of artificially assuming an upper bound or inequality scaling, the minimax theory is used to treat the external unknown disturbances. The system is insensitive to effects of large disturbances due to taking into account the worst case disturbance. Second, a parameter projection mechanism is introduced in adaptive control to force the parameter estimate within a prior specified interval. The proposed controller handles the nonlinear parameterization without compromising control smoothness and at the same time the parameter estimate speed is improved and the robustness of system is strengthened. Considering the short-circuit ground fault and mechanical power perturbation, a simulation study is carried out. The results show the effectiveness of the proposed control method.
\end{abstract}

\section{Introduction}

With the expansion of the scale of electric network, static var compensators (SVCs) have been employed in power systems for several years in a cost-effective manner [1]. SVCs play important roles in voltage regulation and stability improvement due to simple structures and reactive power compensation [2]. Numerous control techniques with varying levels of success for SVC have been used to enhance power system stability $[3,4]$. The fixed-gain PID controllers are designed for improving the dynamic impact of SVCs based on the linearization model without taking nonlinear characteristic into consideration [5]. The exact feedback linearization design depends on the basis of nonlinear SVC model [6]; however, such a solution requires a completely accurate model, which is rarely satisfying from the practical point of view. The Hamiltonian function method cannot only develop nonlinear control for the SVC, but also solve the problem of $L_{2}$ disturbance attenuation [7], whereas it is hard work to express the nonlinear system into a Hamiltonian system. Adaptive backstepping technique has received a considerable attention in recent robust control literatures of power systems $[8,9]$.
Several papers have studied the adaptive backstepping SVC control strategies and gave insights into the effect of external disturbance. There are many causes of variations in a power system's operating conditions, such as continual changes in power consumption and changes in the generation and transmission device structure. Significant progress has been made in disturbance treatment linking with backstepping method; the $H_{\infty}$ control problem can be solved by inequality-scaling the item including disturbance in energy function $[10,11]$, while the scaling way may have brought conservativeness. Although many works successfully deal with the disturbances, the disturbances are always restricted with a certain bound or a certain expression [12-14]. The upper bound is difficult to be selected because of the difficulty of exact measurement in some practical applications [15]. It is the objective of this paper to provide an effective way in unknown disturbance treatment to overcome the above disadvantages. The minimax method is an efficient approach to deal with large disturbance attenuation problem by estimating the degree of damage [16, 17]; an in-depth study on the large disturbance attenuation problems of the nonlinear TCSC and STATCOM is conducted via adaptive backstepping and minimax method $[18,19]$. 
References [18, 19] also play a key role in uncertain parameter estimation. However, conventional adaptive controls always ignore the available prior information of the uncertainties, which may lead to poor and slow convergence, because the parameter search process possibly takes place outside the region of true value. It is often reasonable to obtain the knowledge on bounds of unknown parameter of the SVC model. To absorb the prior information, the projection technique can be adopted [20,21]. A novel adaptive control solution is provided; this approach enforces prior known upper and lower bounds of the uncertain parameters always on their corresponding estimates, without compromising control smoothness or global stability guarantees for the closed-loop dynamics [22].

This paper addresses the nonlinear robust control problem for the SVC system with unknown external disturbances and parameter uncertainties using modified adaptive backstepping and minimax approach. In order to avoid the conservativeness brought by conventional disturbance treatment, a test function related to the performance index is constructed to maximize the impact of the disturbances, and the feedback control is investigated by taking account of the worst case. Moreover, the class- $\kappa$ functions are used in the design procedure to keep the balance between transient response and controller gain. For the uncertainties, a projection mechanism is applied depending on the available bounds on the plant parameters, which can promote the efficiency of parameter search process. Compared with traditional adaptive backstepping method, numerical simulations of two kinds of disturbances to the SVC system demonstrate that the proposed control gives superiorities on transient performance.

\section{Dynamic Model and Problem Statements}

Consider the following dynamic model of single-machine infinite-bus (SMIB) power system with SVC as shown in Figure 1 [11].

The mathematical dynamics of SVC control system can be expressed by the following nonlinear differential equations [11]

$$
\begin{gathered}
\dot{\delta}=\omega-\omega_{0}, \\
\dot{\omega}=\frac{\omega_{0}}{H}\left(P_{m}-E_{q}^{\prime} V_{s} y_{\mathrm{svc}} \sin \delta\right)-\frac{D}{H}\left(\omega-\omega_{0}\right), \\
\dot{y}_{\mathrm{svc}}=\frac{1}{T_{\mathrm{svc}}}\left(-y_{\mathrm{svc}}+y_{\mathrm{svc} 0}+u\right) .
\end{gathered}
$$

In the above equations, $\delta$ is the rotor angle; $\omega$ is the angular speed; $H$ and $D$ are the inertia constant and damping coefficient; $P_{m}$ is the mechanical power; $E_{q}^{\prime}$ and $V_{s}$ are the $q$ axis transient reactance and infinite bus voltage; $y_{\text {svc }}$ is the susceptance of the overall system, $y_{\mathrm{svc}}=1 /\left(X_{1}+X_{2}+\right.$ $\left.X_{1} X_{2}\left(B_{L}+B_{C}\right)\right), X_{1}=X_{d}^{\prime}+X_{T}+X_{L}, X_{2}=X_{L}, X_{d}^{\prime}, X_{T}$ and $X_{L}$ are, respectively, the direct axis transient reactance of the generator, the reactance of the transformer, and the line reactance, and $B_{L}$ and $B_{C}$ are the susceptance of the inductor

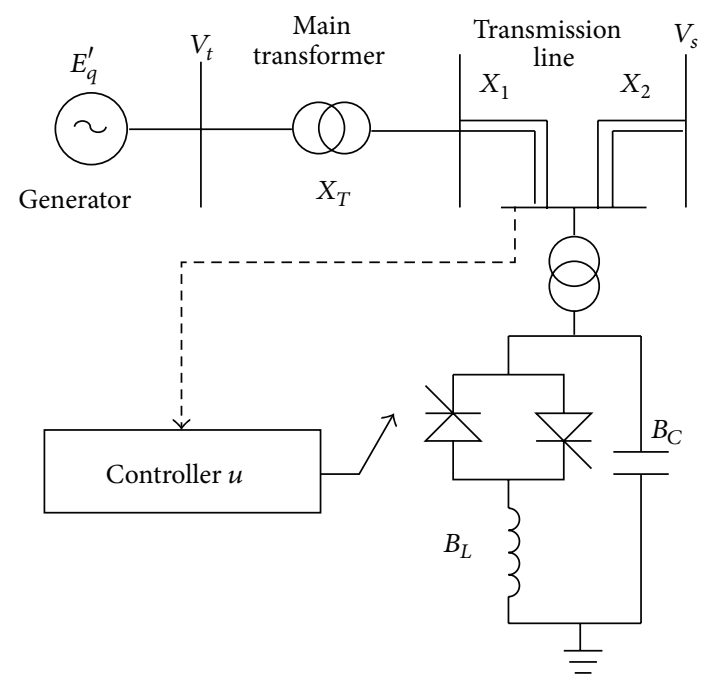

FIGURE 1: Single machine infinite bus system with SVC.

and the capacitor in SVC; $T_{\text {svc }}$ is the time constant of SVC; $u$ is the equivalence input of SVC regulator.

Denote $x_{1}=\delta-\delta_{0}, x_{2}=\omega-\omega_{0}$, and $x_{3}=y_{\mathrm{svc}}-y_{\mathrm{svc} 0}$, where $\left(\delta_{0}, \omega_{0}, y_{\mathrm{svc} 0}\right)$ represent an operating point of the power system. Consider the external disturbance vector $\varepsilon=$ $\left[\begin{array}{ll}\varepsilon_{1} & \varepsilon_{2}\end{array}\right]^{T} ; \varepsilon_{1}$ and $\varepsilon_{2}$ are unknown functions that belong to $L_{2}$ space. Then, system (1) can be transformed into the following form:

$$
\dot{x}_{1}=x_{2} \text {, }
$$

$$
\dot{x}_{2}=\theta x_{2}+a_{0} P_{m}+b_{0}\left(x_{3}+y_{\text {svc } 0}\right) \sin \left(x_{1}+\delta_{0}\right)+\varepsilon_{1},
$$

$$
\dot{x}_{3}=-m_{0} x_{3}+m_{0} u+\varepsilon_{2} \text {, }
$$

where $a_{0}=\omega_{0} / H, b_{0}=-\omega_{0} E_{q}^{\prime} V_{s} / H, m_{0}=1 / T_{\text {svc }}$. Let $\theta=-D / H$ be an uncertain constant parameter in view of the damping coefficient $D$ that cannot be measured accurately. However, it is reasonable to obtain a prior knowledge on its bound, both from literatures and practice [23-25]. Hence, the upper and lower bounds of $\theta$ can also be acquired; we assume $\theta \in\left(\theta_{\min }, \theta_{\max }\right)$.

\section{Adaptive Disturbance Attenuation Design for SVC Control System}

In the control of large scale power system, one usually faces limited knowledge on plant parameters and the appearance of sudden large disturbances. A well-designed controller should have the ability to perform its desired function in the presence of changes and uncertainties in the system. The proposed approach is aiming to attenuate the external disturbance and estimate the uncertainty. We adopt the minimax method and parameter projection mechanism based on backstepping technique to deal with the problems. 
Step 1. Start with (2a); we define $e_{1}=x_{1}$ and view $x_{2}$ as a control variable. Design a virtual control law $x_{2}^{*}$ as $x_{2}^{*}=-\left[c_{1}+\right.$ $\left.\varphi_{1}\left(\left|e_{1}\right|\right)\right] e_{1}$, where $c_{1}>0$, and $\varphi_{1}(\cdot)$ is a class- $\kappa$ function; we select $\varphi_{1}\left(\left|e_{1}\right|\right)=k_{1} e_{1}^{2}, k_{1}>0$. Define an error variable $e_{2}=$ $x_{2}-x_{2}^{*}$ representing the difference between the actual and virtual controls. Then, we can derive the dynamics of the new coordinate

$$
\dot{e}_{1}=-\left[c_{1}+\varphi_{1}\left(\left|e_{1}\right|\right)\right] e_{1}+e_{2}
$$

The objective of this step is to make $e_{1} \rightarrow 0$, by considering the Lyapunov function as

$$
V_{1}=\frac{\sigma}{2} e_{1}^{2}
$$

where $\sigma>0$; then the time derivative of $V_{1}$ becomes

$$
\dot{V}_{1}=-\sigma c_{1} e_{1}^{2}-\sigma \varphi_{1}\left(\left|e_{1}\right|\right) e_{1}^{2}+\sigma e_{1} e_{2}
$$

Apparently, if $e_{2}=0$, then $\dot{V}_{1}=-\sigma c_{1} e_{1}^{2}-\sigma k_{1} e_{1}^{4} \leq 0$, and $e_{1}$ is guaranteed to converge to zero asymptotically. The coupling term $\sigma e_{1} e_{2}$ will be canceled in the next step.

Step 2. Consider (2b) by viewing $x_{3}$ as a virtual control variable. Define a virtual control law $x_{3}^{*}$ and the error variable $e_{3}=x_{3}-x_{3}^{*}$. Our objective in this step is to make $e_{2} \rightarrow 0$, and then choose a Lyapunov function by augmenting (4):

$$
V_{2}=V_{1}+\frac{1}{2} e_{2}^{2}
$$

Before virtual control law design, we plot out a regulated output $z=\left[\begin{array}{ll}q_{1} e_{1} & q_{2} e_{2}\end{array}\right]^{T}$ into system (2a), (2b), and (2c), where $q_{1}$ and $q_{2}$ are nonnegative weighted coefficients representing weighting proportion of $e_{1}$ and $e_{2}$. Then, construct a performance index based on minimax theory as

$$
J_{1}=\int_{0}^{\infty}\left(\|z\|^{2}-\gamma^{2}\left\|\varepsilon_{1}\right\|^{2}\right) d t
$$

where $\gamma>0$, and $\gamma$ is disturbance attenuation constant. Further, construct a test function related to the performance index to estimate the worst case disturbance, which means the highest degree of critical disturbance that can be endured by the system:

$$
H_{1}=\dot{V}_{2}+\frac{1}{2}\left(\|z\|^{2}-\gamma^{2}\left\|\varepsilon_{1}\right\|^{2}\right)
$$

Substituting $\dot{V}_{2}=\dot{V}_{1}+e_{2} \dot{e}_{2}$ into (8) yields

$$
\begin{aligned}
H_{1}= & -\sigma c_{1} e_{1}^{2}-\sigma k_{1} e_{1}^{4}+\sigma e_{1} e_{2} \\
& +e_{2}\left(\theta x_{2}+a_{0} P_{m}+b_{0}\left(x_{3}+y_{\mathrm{svc} 0}\right) \sin \left(x_{1}+\delta_{0}\right)\right. \\
& \left.+\varepsilon_{1}+c_{1} x_{2}+3 k_{1} x_{1}^{2} x_{2}\right) \\
& +\frac{1}{2} q_{1}^{2} e_{1}^{2}+\frac{1}{2} q_{2}^{2} e_{2}^{2}-\frac{1}{2} \gamma^{2} \varepsilon_{1}^{2} .
\end{aligned}
$$

We assume that the upper value of (7) is $J_{1}^{*}$. If a disturbance exists and makes $J_{1}$ no larger than $J_{1}^{*}$, then the degree of damage is greatest on the system performance. Thus, our task here is to maximize $J_{1}$ by making the firstorder derivative of $H_{1}$ with respect to $\varepsilon_{1}$ equal to zero, which is equivalent to $e_{2}-\gamma^{2} \varepsilon_{1}=0$; then we derive

$$
\varepsilon_{1}^{*}=\frac{e_{2}}{\gamma^{2}}
$$

Furthermore, we compute the second-order derivative; that is, $\partial^{2} H_{1} / \partial \varepsilon_{1}^{2}=-\gamma^{2}<0$. Therefore, the maximum value of $H_{1}$ about $\varepsilon_{1}$ exists, and

$$
\max H_{1}=\max \left[\dot{V}_{2}+\frac{1}{2}\left(\|z\|^{2}-\gamma^{2}\left\|\varepsilon_{1}\right\|^{2}\right)\right] .
$$

Integrating both sides of (11) yields

$$
\begin{aligned}
\max & \int_{0}^{\infty} H_{1} d t \\
& =\max \left[\int_{0}^{\infty} \dot{V}_{2} d t+\frac{1}{2} \int_{0}^{\infty}\left(\|z\|^{2}-\gamma^{2}\left\|\varepsilon_{1}\right\|^{2}\right) d t\right] .
\end{aligned}
$$

Let $\bar{H}_{1}=\int_{0}^{\infty} H_{1} d t$; then (12) becomes $\max \bar{H}_{1}=$ $\max \left[V_{2}(\infty)-V_{2}(0)+(1 / 2) J_{1}\right]$, and then

$$
\max \left(\frac{1}{2} J_{1}\right)=\max \left(\bar{H}_{1}-\Delta V_{2}\right) \leq \max \left(\bar{H}_{1}\right)-\min \left(\Delta V_{2}\right) .
$$

When the system suffers sufficiently large disturbances, $V_{2}$ will not be reduced, in other words, the disturbance $\varepsilon_{1}$ is assumed to reduce $V_{2}$ to 0 ; that is, $\min \left(\Delta V_{2}\right)=0$. Thus, it proves that $\max \left((1 / 2) J_{1}\right)=\max \left(\bar{H}_{1}\right)$, and $\varepsilon_{1}^{*}$ is the worst case disturbance for the subsystem.

Remark 1. From the equivalent analysis of $\max \left(\bar{H}_{1}\right)$ and $\max \left((1 / 2) J_{1}\right)$, it is obvious that if $\varepsilon_{1}$ allows $\bar{H}_{1}$ to obtain the maximum value, $\varepsilon_{1}$ also allows $J_{1}$ to obtain the maximum value. That is, system performance damage via $\varepsilon_{1}$ is the largest.

The stabilizing function $x_{3}^{*}$ needs to be designed by undertaking the disturbances with such damage degree into system; our approach is to replace $\varepsilon_{1}$ in (9) with (10):

$$
\begin{aligned}
H_{1}= & -\sigma c_{1} e_{1}^{2}-\sigma k_{1} e_{1}^{4}+\sigma e_{1} e_{2} \\
& +e_{2}\left(\theta x_{2}+a_{0} P_{m}+b_{0}\left(x_{3}+y_{\mathrm{svc} 0}\right) \sin \left(x_{1}+\delta_{0}\right)\right. \\
& \left.+\frac{e_{2}}{\gamma^{2}}+c_{1} x_{2}+3 k_{1} x_{1}^{2} x_{2}\right) \\
+ & \frac{1}{2} q_{1}^{2} e_{1}^{2}+\frac{1}{2} q_{2}^{2} e_{2}^{2}-\frac{1}{2} \gamma^{2}\left(\frac{e_{2}}{\gamma^{2}}\right)^{2} .
\end{aligned}
$$




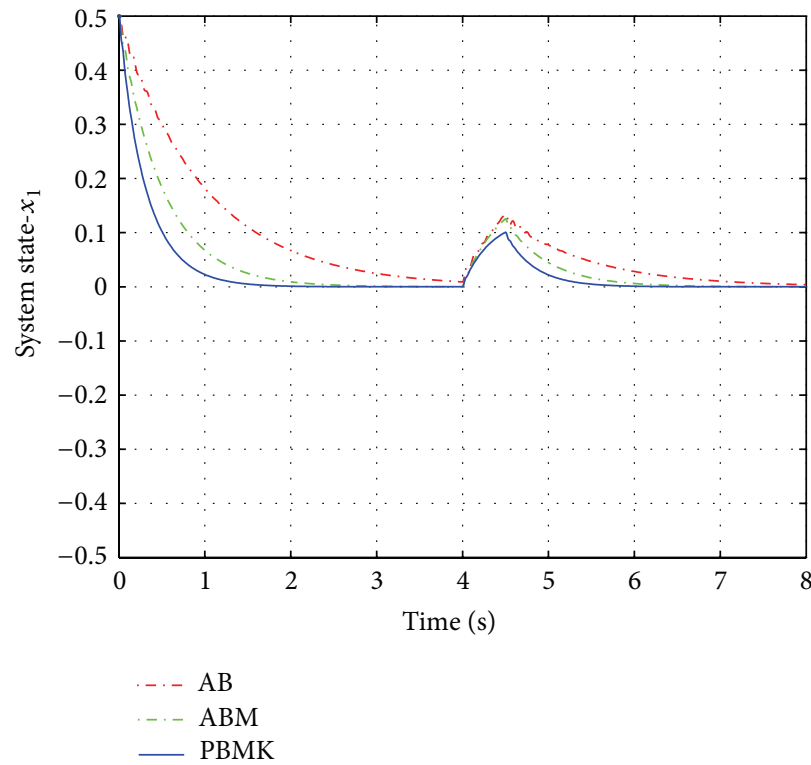

(a) The curves of the angle

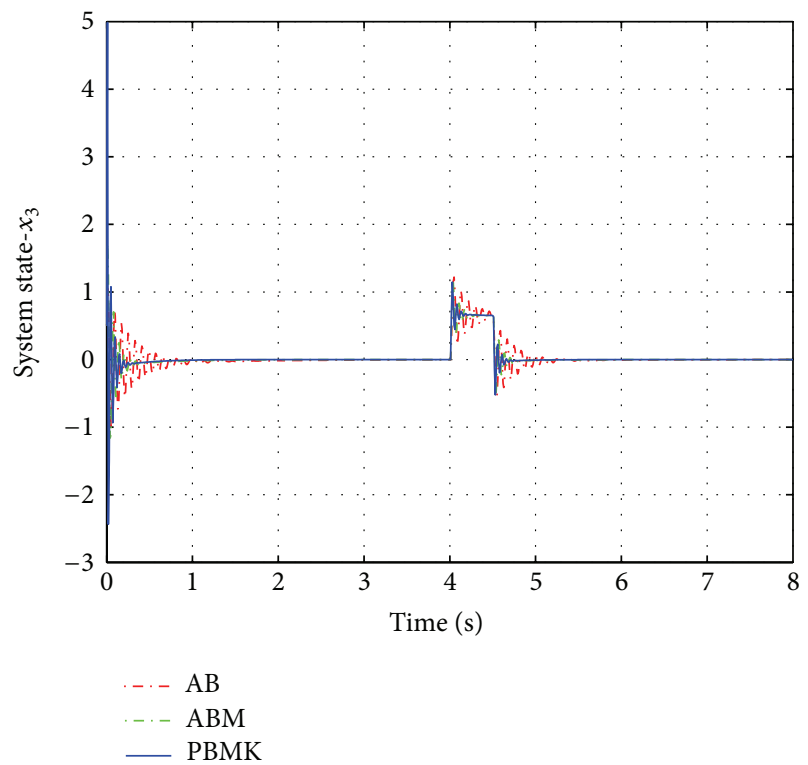

(c) The curves of the susceptance

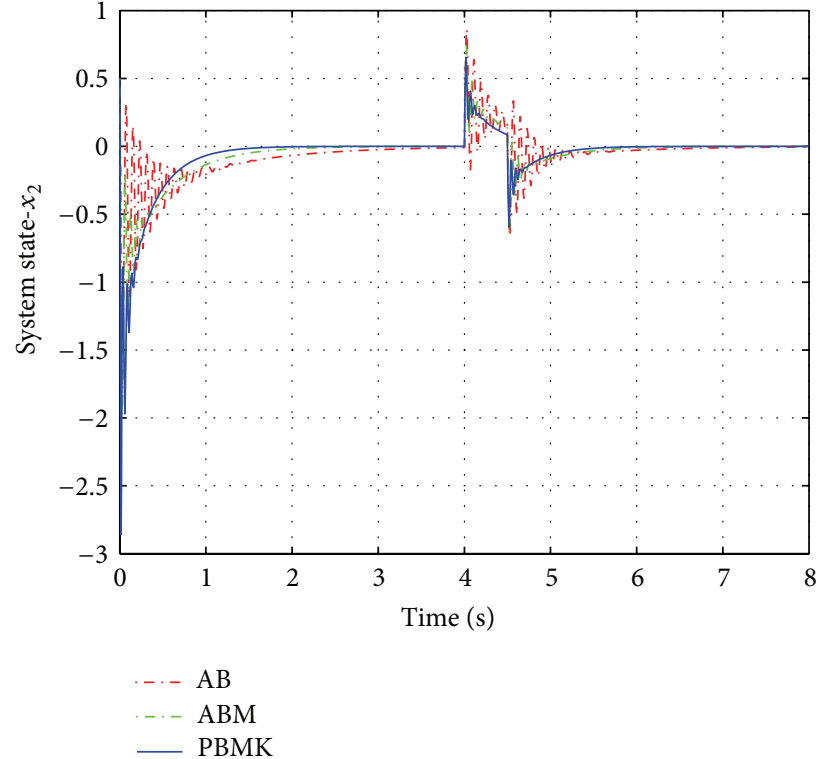

(b) The curves of the relative speed

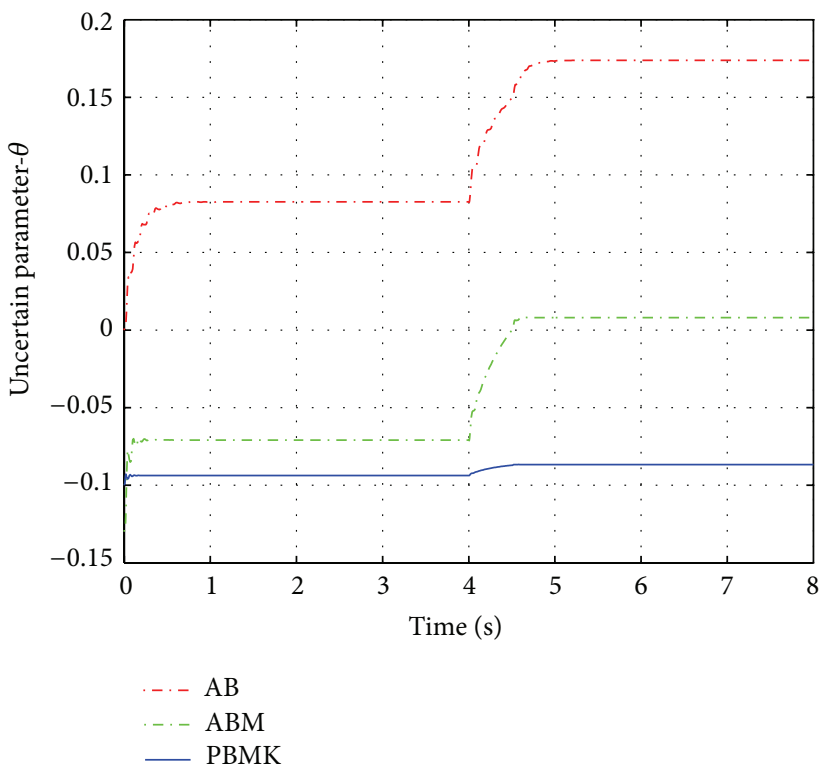

(d) The curves of the parameter estimation

FIGURE 2: Transient response of closed-loop system under the short circuit ground fault.

Suppose that $h_{1}=\sigma c_{1}-(1 / 2) q_{1}^{2} ; h_{2}=c_{2}+(1 / 2) q_{2}^{2}+1 / 2 \gamma^{2}$, $c_{2}>0 ; f_{\sin }=\sin \left(x_{1}+\delta_{0}\right)$. Now we select

$$
\begin{aligned}
x_{3}^{*}=-\frac{1}{b_{0} f_{\mathrm{sin}}}[ & h_{2} e_{2}+\varphi_{2}\left(\left|e_{2}\right|\right) e_{2}+\sigma e_{1}+\widehat{\theta} x_{2} \\
& \left.+c_{1} x_{2}+a_{0} P_{m}+3 k_{1} x_{1}^{2} x_{2}\right]-y_{\mathrm{svc} 0},
\end{aligned}
$$

where $\varphi_{2}(\cdot)$ is a class- $\kappa$ function; we choose $\varphi_{2}\left(\left|e_{2}\right|\right)=k_{2} e_{2}^{2}$, $k_{2}>0 ; \widetilde{\theta}$ is an estimate of $\theta$, and $\widetilde{\theta}=\theta-\widehat{\theta}$. If the rotor angle $\delta=k \pi, k=0,1, \ldots$, synchronism of the power system will be lost. Fortunately, under the normal operating conditions in the system $0<\delta<\pi$ holds, and therefore, the condition $\sin \left(x_{1}+\delta_{0}\right) \neq 0$ can be guaranteed.
Then, $H_{1}=-h_{1} e_{1}^{2}-\sigma k_{1} e_{1}^{4}-c_{2} e_{2}^{2}-k_{2} e_{2}^{4}+e_{2} \tilde{\theta} x_{2}+b_{0} e_{2} e_{3} f_{\text {sin }}$. In the final step, the coupling term $b_{0} e_{2} e_{3} f_{\text {sin }}$ will be canceled, and the uncertainty item $e_{2} \widetilde{\theta} x_{2}$ will be dealt with.

Step 3. For the uncertainty, as mentioned in Section 2, it is reasonable to expect availability of a prior knowledge in terms of lower and upper bounds of $\theta$ in (2a), (2b), and (2c). Thereby, we reparameterize the uncertain parameter $\theta$ in an associated uncertain variable $\phi$ as follows [22]:

$$
\theta=\frac{1}{2}\left(\theta_{\max }-\theta_{\min }\right)(1-\tanh \phi)+\theta_{\min } .
$$




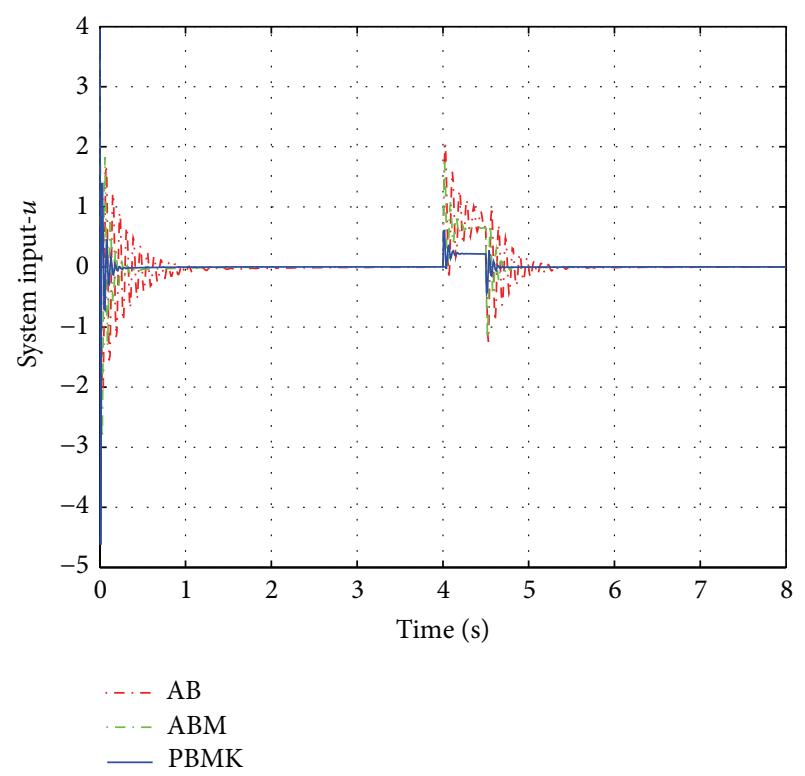

FIgURE 3: Control input curves under the short circuit ground fault.

It is clear that is for all values of $\phi \in R, \tanh \phi \in$ $(-1,1)$, hence, $\theta$ is restricted to lie within the region of $\left(\theta_{\min }, \theta_{\max }\right)$. Consequently, the system governing equation in (2b), which is linear in terms of $\theta$, immediately becomes nonlinear in terms of $\phi$. We are in the position to develop a smooth adaptive controller in order to handle the nonlinear parameterization of (16). We define $z=\widehat{\phi}-\phi$, wherein $\widehat{\phi}$ is the estimate of $\phi$; then choose the following nonnegative, and therefore, lower-bounded function of $z$ and $\phi$ as

$$
V_{z}=\frac{1}{2}\left(\theta_{\max }-\theta_{\min }\right)[\ln \cosh (z+\phi)-z \tanh \phi] .
$$

Consider the candidate Lyapunov function as

$$
V_{3}=V_{2}+\frac{1}{2} e_{3}^{2}+\frac{1}{\rho} V_{z}
$$

The time derivative of $V_{3}$ becomes

$$
\dot{V}_{3}=\dot{V}_{2}+e_{3} \dot{e}_{3}+\frac{1}{2 \rho}\left(\theta_{\max }-\theta_{\min }\right)[\tanh (z+\phi)-\tanh \phi] \dot{z}
$$

The performance index is expressed as

$$
J_{2}=\int_{0}^{\infty}\left(\|z\|^{2}-\gamma^{2}\|\varepsilon\|^{2}\right) d t
$$

The test function is

$$
H_{2}=\dot{V}_{3}+\frac{1}{2}\left(\|z\|^{2}-\gamma^{2}\|\varepsilon\|^{2}\right) .
$$

Substituting (19) into (21) yields

$$
\begin{aligned}
& H_{2}=-h_{1} e_{1}^{2}-\sigma k_{1} e_{1}^{4}-c_{2} e_{2}^{2}-k_{2} e_{2}^{4}+e_{2} \tilde{\theta} x_{2}+b_{0} e_{2} e_{3} f_{\text {sin }} \\
& +e_{3}\left\{-m_{0} x_{3}+m_{0} u+\varepsilon_{2}+\frac{1}{b_{0} f_{\text {sin }}}\right. \\
& \times\left[(\sigma+\dot{\hat{\theta}}) x_{2}+6 k_{1} x_{1} x_{2}^{2}\right. \\
& +\left(h_{2}+3 k_{2} e_{2}^{2}\right)\left(c_{1} x_{2}+3 k_{1} e_{1}^{2} x_{2}\right) \\
& +\left(\widehat{\theta}+c_{1}+3 k_{1} x_{1}^{2}+h_{2}+3 k_{2} e_{2}^{2}\right) \\
& \left.\times\left(\theta x_{2}+a_{0} P_{m}+b_{0}\left(x_{3}+y_{\mathrm{svc} 0}\right) f_{\text {sin }}+\frac{e_{2}}{\gamma^{2}}\right)\right] \\
& -\frac{f_{\cos } x_{2}}{b_{0} f_{\sin }^{2}}\left(h_{2} e_{2}+k_{2} e_{2}^{3}+\sigma e_{1}+\hat{\theta} x_{2}\right. \\
& \left.\left.+c_{1} x_{2}+a_{0} P_{m}+3 k_{1} x_{1}^{2} x_{2}\right)\right\} \\
& -\frac{1}{2} \gamma^{2} \varepsilon_{2}^{2}+\frac{1}{2 \rho}\left(\theta_{\max }-\theta_{\min }\right) \\
& \times[\tanh (z+\phi)-\tanh \phi] \dot{z},
\end{aligned}
$$

where $f_{\cos }=\cos \left(x_{1}+\delta_{0}\right)$. A similar procedure is employed to make $\partial \mathrm{H}_{2} / \partial \varepsilon_{2}=0$; we can obtain the worst case disturbance $\left(\partial^{2} \mathrm{H}_{2} / \partial \varepsilon_{2}^{2}=-\gamma^{2}<0\right)$

$$
\varepsilon_{2}^{*}=\frac{e_{3}}{\gamma^{2}} .
$$

Taking (23) into account, (22) is rewritten as

$$
\begin{aligned}
H_{2}=-h_{1} e_{1}^{2}-\sigma k_{1} e_{1}^{4} & -c_{2} e_{2}^{2}-k_{2} e_{2}^{4} \\
+e_{3}\left\{b_{0} e_{2} f_{\sin }\right. & -m_{0} x_{3}+m_{0} u+\frac{e_{3}}{2 \gamma^{2}} \\
+\frac{1}{b_{0} f_{\text {sin }}} & {\left[(\sigma+\dot{\hat{\theta}}) x_{2}+6 k_{1} x_{1} x_{2}^{2}\right.} \\
+ & \left(h_{2}+3 k_{2} e_{2}^{2}\right)\left(c_{1} x_{2}+3 k_{1} e_{1}^{2} x_{2}\right) \\
+ & \left(\widehat{\theta}+c_{1}+3 k_{1} x_{1}^{2}+h_{2}+3 k_{2} e_{2}^{2}\right) \\
& \left.\times\left(\widehat{\theta} x_{2}+a_{0} P_{m}+b_{0}\left(x_{3}+y_{\mathrm{svc} 0}\right) f_{\sin }+\frac{e_{2}}{\gamma^{2}}\right)\right] \\
-\frac{f_{\cos } x_{2}}{b_{0} f_{\text {sin }}^{2}} & \left(h_{2} e_{2}+k_{2} e_{2}^{3}+\sigma e_{1}+\hat{\theta} x_{2}\right. \\
& \left.\left.+c_{1} x_{2}+a_{0} P_{m}+3 k_{1} x_{1}^{2} x_{2}\right)\right\}
\end{aligned}
$$




$$
\begin{aligned}
& +\left[e_{2} x_{2}+\frac{e_{3} x_{2}}{b_{0} f_{\text {sin }}}\left(\hat{\theta}+c_{1}+3 k_{1} x_{1}^{2}+h_{2}+3 k_{2} e_{2}\right)\right] \tilde{\theta} \\
& +\frac{1}{2 \rho}\left(\theta_{\max }-\theta_{\min }\right)[\tanh (z+\phi)-\tanh \phi] \dot{z},
\end{aligned}
$$

in which $\widehat{\theta}=(1 / 2)\left(\theta_{\max }-\theta_{\min }\right)(1-\tanh \widehat{\phi})+\theta_{\min }$, and then $\tilde{\theta}=(1 / 2)\left(\theta_{\max }-\theta_{\min }\right)(\tanh \widehat{\phi}-\tanh \phi)$.

For the purpose of making $\mathrm{H}_{2} \leq 0$, we select an adaptive controller consisting of an actual control input $u$ and a reparameter estimator, which provides the estimate of $\phi$ :

$$
\begin{aligned}
& u=-\frac{1}{m_{0}} \\
& \times\left\{\left(c_{3}+\frac{1}{2 \gamma^{2}}+\varphi_{3}\left(\left|e_{3}\right|\right)\right) e_{3}\right. \\
& +\frac{1}{b_{0} f_{\text {sin }}}\left[\left(\sigma-\frac{1}{2}\left(\theta_{\max }-\theta_{\text {min }}\right) \frac{\dot{\hat{\phi}}}{\cosh ^{2} \widehat{\phi}}\right) x_{2}\right. \\
& +6 k_{1} x_{1} x_{2}^{2}+\left(h_{2}+3 k_{2} e_{2}^{2}\right)\left(c_{1} x_{2}+3 k_{1} e_{1}^{2} x_{2}\right) \\
& +\left(\hat{\theta}+c_{1}+3 k_{1} x_{1}^{2}+h_{2}+3 k_{2} e_{2}^{2}\right) \\
& \left.\times\left(\widehat{\theta} x_{2}+b_{0}\left(x_{3}+y_{\text {svc } 0}\right) f_{\text {sin }}+a_{0} P_{m}+\frac{e_{2}}{\gamma^{2}}\right)\right] \\
& -\frac{f_{\cos } x_{2}}{b_{0} f_{\text {sin }}^{2}}\left(h_{2} e_{2}+k_{2} e_{2}^{3}+\sigma e_{1}+\widehat{\theta} x_{2}\right. \\
& \left.+c_{1} x_{2}+a_{0} P_{m}+3 k_{1} x_{1}^{2} x_{2}\right) \\
& \left.+b_{0} e_{2} f_{\sin }-m_{0} x_{3}\right\} \text {, } \\
& \dot{\hat{\phi}}=-\rho\left[e_{2} x_{2}\right. \\
& +\frac{e_{3} x_{2}}{b_{0} f_{\sin }}\left(\frac{1}{2}\left(\theta_{\max }-\theta_{\min }\right)(1-\tanh \widehat{\phi})\right. \\
& \left.\left.+\theta_{\text {min }}+c_{1}+3 k_{1} x_{1}^{2}+h_{2}+3 k_{2} e_{2}^{2}\right)\right] \text {, }
\end{aligned}
$$

where $\varphi_{3}(\cdot)$ is a class- $\kappa$ function. We choose $\varphi_{3}\left(\left|e_{3}\right|\right)=k_{3} e_{3}^{2}$, $k_{3}>0$, and then $\hat{\phi}$ is generated through the solution of the differential equations governed by (26). And $\widehat{\theta}$, the estimate of $\theta$, is indirectly obtained by $\widehat{\phi}$ :

$$
\widehat{\theta}=\frac{1}{2}\left(\theta_{\max }-\theta_{\min }\right)(1-\tanh \widehat{\phi})+\theta_{\min } .
$$

Then, we can obtain

$$
H_{2}=-h_{1} e_{1}^{2}-\sigma k_{1} e_{1}^{4}-c_{2} e_{2}^{2}-k_{2} e_{2}^{4}-c_{3} e_{3}^{2}-k_{3} e_{3}^{4} \leq 0 .
$$

If we define $V(x)=2 V_{3}(x)$ as the overall Lyapunov function, then it follows readily that

$$
\dot{V} \leq \gamma^{2}\|\varepsilon\|^{2}-\|z\|^{2}
$$

Equation (29) indicates that all increased energy of SVC system from $t=0$ to any final time is always smaller than or equal to the ones from outside; that is, the system energy is decreasing.

Theorem 2. For the given disturbance attenuation constant $\gamma>0$, the $L_{2}$ disturbance attenuation problem of system (1) can be solved by adaptive controller (25) to (27), and a positive storage function $V(x)$ exists such that the dissipation inequality

$$
V(x(t))-V(x(0)) \leq \int_{0}^{T}\left(\gamma^{2}\|\varepsilon\|^{2}-\|z\|^{2}\right) d t
$$

holds for any final time $T$, and the closed-loop system is characteristic with disturbance rejection.

When $\varepsilon_{1}=0, \varepsilon_{2}=0$, the closed-loop system is asymptotically stable. When $\varepsilon_{1} \neq 0, \varepsilon_{2} \neq 0$, the $L_{2}$ gain from the disturbance to the output of the system is smaller than or equal to $\gamma$. According to the definition of virtual control, the $x_{1}, x_{2}$, and $x_{3}$ are bounded convergences.

Remark 3. The class- $\kappa$ function $\varphi_{i}(\cdot)$ is introduced into the selection of stabilizing function $x_{i}^{*}, i=1,2,3$, during the recursive design procedure, in order to keep the balance between transient response and controller gain. This approach promotes convergent speed remarkably without increasing the controller gain.

Remark 4. Exist disturbance treatment usually assumes the plant with bounded disturbance or zooms the items of the energy function about the disturbance, which probably increase the conservativeness. This paper adopts the minimax method to maximize the effects of disturbances. The control law is designed by undertaking the worst case disturbance to ensure the stability of the closed-loop system. Thus, the system is theoretically not sensitive to disturbance effects.

Remark 5. Different from the previous adaptive method in power systems, we fully and properly utilize all the available prior information on the bound of unknown parameter by adopting parameter projection technique. We select a specific uncertain parameter structure to force the parameter estimate to stay within the valid region and generate a smooth adaptive control law. Accordingly, the transient performance is significantly improved.

\section{Results and Discussion}

We will consider two kinds of disturbances in the digital simulation for the single-machine infinite-bus system with SVC. The physical parameters are selected as follows: $H=$ $5.9 \mathrm{~s}, D=1.0, E_{q}^{\prime}=1.123 \mathrm{pu}, V_{\mathrm{s}}=1.0 \mathrm{pu}, T_{\mathrm{svs}}=0.02 \mathrm{~s}$, $X_{1}=0.84 \mathrm{pu}, X_{2}=0.52 \mathrm{pu}$, and $B_{L}+B_{C}=0.3 \mathrm{pu}$. The operating point is $\delta_{0}=0.9 \mathrm{rad}, \omega_{0}=314.159 \mathrm{rad} / \mathrm{s}$, and 


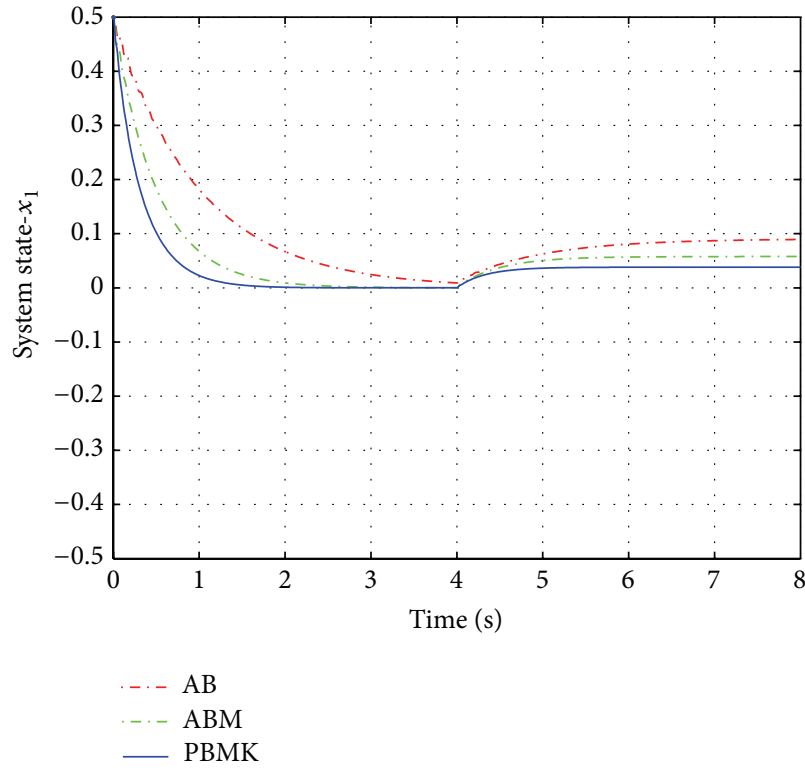

(a) The curves of the angle

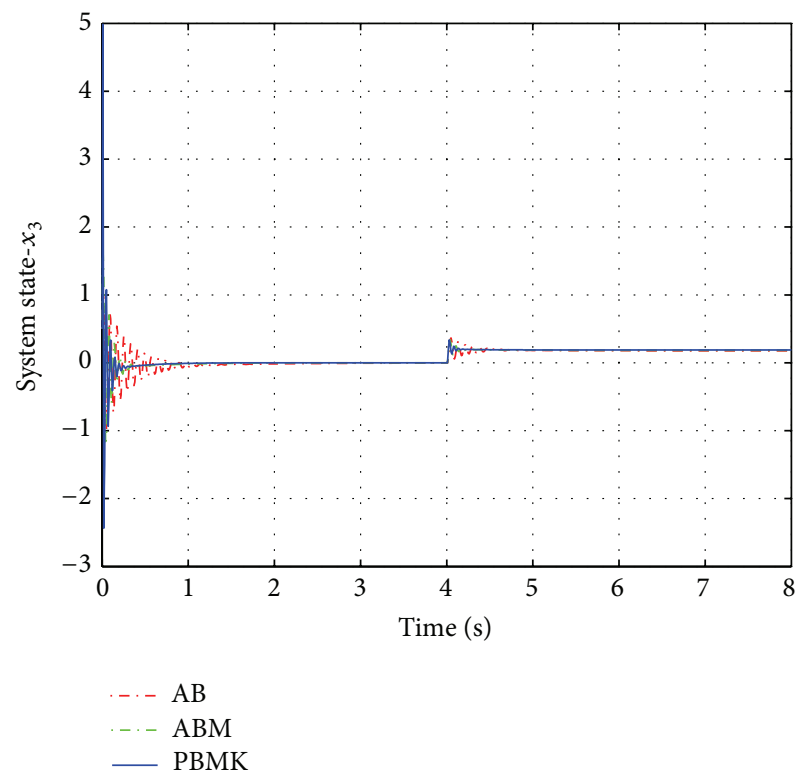

(c) The curves of the susceptance

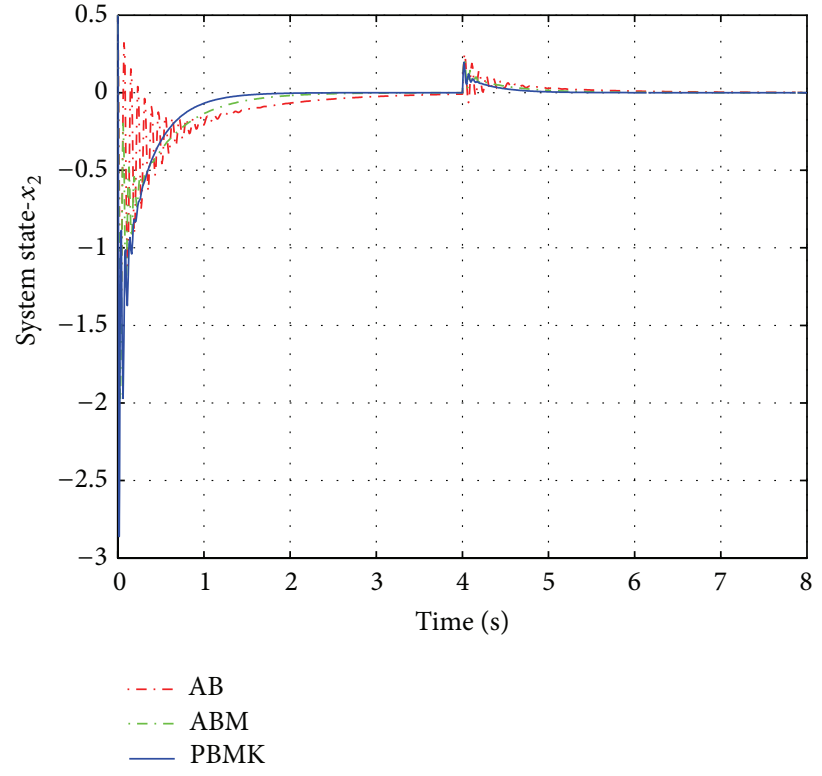

(b) The curves of the relative speed

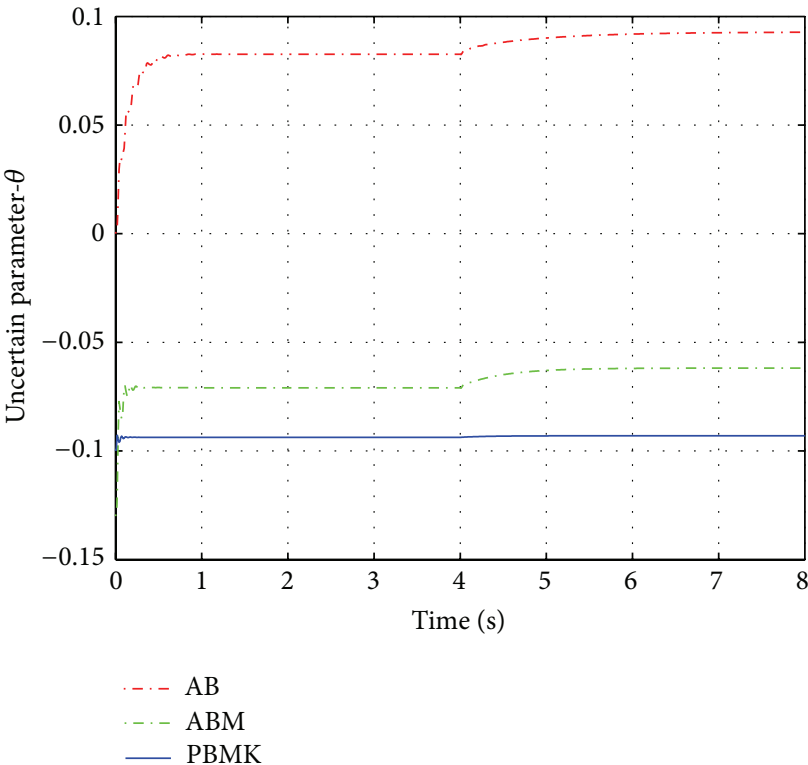

(d) The curves of the parameter estimation

Figure 4: Transient response of closed-loop system under the mechanical power perturbation.

$y_{\text {svc0 }}=0.4 \mathrm{pu}$. The control parameters are selected as follows: $q_{1}=0.4, q_{2}=0.6, c_{1}=2, c_{2}=2, c_{3}=2, k_{1}=1, k_{2}=1, k_{3}=1$, $\gamma=0.2$, and $\rho=1$. The upper and lower bound of uncertain parameter are $\theta_{\max }=0$ and $\theta_{\min }=-0.5$.

In order to show the effectiveness of the proposed modified parameter projection adaptive backstepping minimax (PBMK) controller, we will make comparisons with the adaptive backstepping minimax (ABM) controller [18] and the conventional adaptive backstepping $(\mathrm{AB})$ controller [10] under the same nonzero initial condition. Note that the control parameters for $\mathrm{ABM}$ controller and $\mathrm{AB}$ controller are selected as $c_{1}=3, c_{2}=3$, and $c_{3}=3$.
4.1. Short Circuit Ground Fault. In $4 \mathrm{~s}$, a transient three-phase short-circuit fault occurred on the transmission line. In $4.5 \mathrm{~s}$, the fault disappears, and the system restores to the normal structure. The reactance of the transformer varies in different stages after a short circuit ground fault as follows:

the period of pre-fault $X_{L}=0.52 \mathrm{pu}$;

the period of fault procedure $X_{L}=\infty$;

the period of after-fault $X_{L}=0.52 \mathrm{pu}$;

the transient response curves of the system are shown in Figures 2 and 3. 


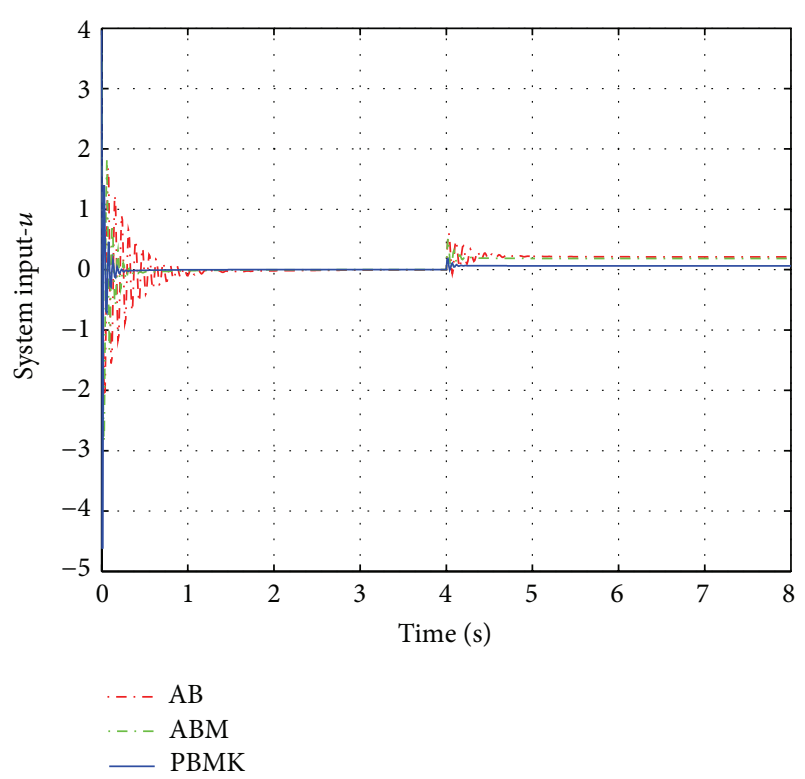

FIGURE 5: Control input curves under the mechanical power perturbation.

Figures 2(a)-2(c) show that, under the proposed controller PBMK, the response is improved without remarkably increasing the controller gain; the convergent speed is faster; the system reaches the stable state more rapidly than the ones under $\mathrm{ABM}$ and $\mathrm{AB}$ controller. Moreover, $\mathrm{PBMK}$ and $\mathrm{ABM}$ controllers, which are both designed by minimax method, have advantages in the ability of disturbance attenuation. Figure 2(d) shows that the proposed adaptive control in this paper ensures that the estimates of the uncertain parameter are always within the prior bounds, while for the ABM and $\mathrm{AB}$ controllers, the parameter search process takes place outside the feasible region where the corresponding "true" parameters lie.

Figure 3 shows that, under PBMK controller, the control input requires bigger energy in the initial period, but it reaches the stable state in short time, and the amplitude of oscillation is relatively smaller. The selected class- $\kappa$ functions converge to zero along with the convergence to zero of the errors. Then the control energy also coincides with that of ABM controller.

4.2. Mechanical Power Perturbation. Unrecoverable mechanical power perturbation occurs at $4 \mathrm{~s}$, and the mechanical power $P_{m}$ changes to another value; that is,

$$
P_{m}= \begin{cases}0.9, & 0 \leq t<4.0 \mathrm{~s} \\ 0.9+\Delta P_{m}, & 4.0 \mathrm{~s}<t, \quad \Delta P_{m}(t)=30 \% P_{m}\end{cases}
$$

The dynamic responses of closed-loop system are shown in Figures 4 and 5.

Figures 4 and 5 show that, after the presence of mechanical power perturbation, the states are stable in a new equilibrium point. And the proposed PBMK controller on the convergence time and the amplitude of oscillation still has advantages compared with $\mathrm{ABM}$ and $\mathrm{AB}$ controllers. The dynamic response of the system does not change significantly with the variety of disturbance form. Therefore, the controller is insensitive to the change in disturbance.

\section{Conclusions}

In this paper, we present an improved robust disturbance attenuation scheme for the nonlinear uncertain SVC system based on improved adaptive backstepping and minimax method. The proposed control strategy gives some advantages, such as the following. (a) The nonlinearities of the SVC system model are completely retained for no linearization process is put on the original system. (b) Our disturbance treatment does not inquire artificially imposing an upper bound on the disturbance or unequally scaling the disturbance items existing in the energy function; then the conservativeness is greatly reduced. (c) The closed-loop system is insensitive to the disturbances because of taking account of the maximum effect of the damage. (d) The class- $\kappa$ functions introduced into the backstepping procedure are helpful to speed up the response without significantly increasing the control gain. (e) We develop a nonlinear smooth function to map the uncertain parameter $\theta$ into $\phi$ in order to restrict $\theta$ to be lying within the prior specified interval, and guarantee that the parameter estimate has a higher convergence rate. Simulation research is under two disturbances that; the results indicate that the proposed control strategy has advantages in terms of the convergence time and oscillation amplitude in comparison with traditional adaptive backstepping approach.

\section{Acknowledgments}

This work is supported by the National Natural Science Foundation of China, under Grants 61304021, 61233002, and 61104074, and the Fundamental Research Funds for the Central Universities, under Grants N110404032 and N110417005.

\section{References}

[1] P. Pourbeik, A. Boström, and B. Ray, "Modeling and application studies for a modern static VAr system installation," IEEE Transactions on Power Delivery, vol. 21, no. 1, pp. 368-377, 2006.

[2] L. Wang and D. N. Truong, "Stability enhancement of a power system with a PMSG-based and a DFIG-based offshore wind farm using a SVC with an adaptive-network-based fuzzy inference system," IEEE Transactions on Industrial Electronics, vol. 60, no. 7, pp. 2799-2807, 2013.

[3] S. Robak, "Robust SVC controller design and analysis for uncertain power systems," Control Engineering Practice, vol. 17, no. 11, pp. 1280-1290, 2009.

[4] S. Teleke, T. Abdulahovic, T. Thiringer, and J. Svensson, "Dynamic performance comparison of synchronous condenser and SVC," IEEE Transactions on Power Delivery, vol. 23, no. 3, pp. 1606-1612, 2008. 
[5] J. C. Dermentzoglou and A. D. Karlis, "Development of linear models of static var compensators and design of controllers suitable for enhancing dynamic/transient performance of power systems including wind farms," Electric Power Systems Research, vol. 81, no. 4, pp. 922-929, 2011.

[6] Y. Ruan and J. Wang, "The coordinated control of SVC and excitation of generators in power systems with nonlinear loads," International Journal of Electrical Power and Energy Systems, vol. 27, no. 8, pp. 550-555, 2005.

[7] Y. Sun, Q. Liu, Y. Song, and T. Shen, "PCH models of facts with adaptive $\mathrm{L}_{2}$-gain control part one theory," Automation of Electric Power Systems, vol. 25, no. 15, pp. 1-6, 2001.

[8] M. Krstic, I. Kanellakopoulos, and P. Kokotovic, Nonlinear and Adaptive Control Design, John Wiley \& Sons, New York, NY, USA, 1995.

[9] J. Fu, "Extended backstepping approach for a class of non-linear systems in generalised output feedback canonical form," IET Control Theory and Applications, vol. 3, no. 8, pp. 1023-1032, 2009.

[10] W. Li, S. Liu, and Y. Jing, "Nonlinear robust control based on adaptive backstepping design for static var compensator," in Proceedings of the 23th Chinese Control Conference, pp. 12501253, 2004.

[11] L.-Y. Sun, S. Tong, and Y. Liu, "Adaptive backstepping sliding mode Ho control of static var compensator," IEEE Transactions on Control Systems Technology, vol. 19, no. 5, pp. 1178-1185, 2011.

[12] C.-S. Tseng, "Robust fuzzy filter design for nonlinear systems with persistent bounded disturbances," IEEE Transactions on Systems, Man, and Cybernetics B, vol. 36, no. 4, pp. 940-945, 2006.

[13] Y. Wang, T. Chai, and Y. Zhang, "State observer-based adaptive fuzzy output-feedback control for a class of uncertain nonlinear systems," Information Sciences, vol. 180, no. 24, pp. 5029-5040, 2010.

[14] H. Coral-Enriquez, J. Cortés-Romero, and G. A. Ramos, "Robust active disturbance rejection control approach to maximize energy capture in variable-speed wind turbines," Mathematical Problems in Engineering, vol. 2013, Article ID 396740, 12 pages, 2013.

[15] X. Zhang, L. Sun, K. Zhao, and L. Sun, "Nonlinear speed control for PMSM system using sliding-mode control and disturbance compensation techniques," IEEE Transactions on Power Electronics, vol. 28, no. 3, pp. 1358-1365, 2013.

[16] I. Petersen, V. Ugrinovskii, and A. Savkin, Robust Control Design Using $H_{\infty}$ Methods, Springer, 2000.

[17] K. Ezal, P. V. Kokotović, A. R. Teel, and T. Başar, "Disturbance attenuating output-feedback control of nonlinear systems with local optimality," Automatica, vol. 37, no. 6, pp. 805-817, 2001.

[18] N. Jiang, B. Liu, J. Kang, Y. Jing, and T. Zhang, "The design of nonlinear disturbance attenuation controller for TCSC robust model of power system," Nonlinear Dynamics, vol. 67, no. 3, pp. 1863-1870, 2012.

[19] N. Jiang, S. Li, T. Liu, and X. Dong, "Nonlinear large disturbance attenuation controller design for the power systems with STATCOM," Applied Mathematics and Computation, vol. 219, pp. 10378-10386, 2013.

[20] K. S. Narendra, "Parameter adaptive control—the end...or the beginning?" in Proceedings of the 33rd IEEE Conference on Decision and Control, pp. 2117-2125, 1994.

[21] R. Bakker and A. M. Annaswamy, "Stability and robustness properties of a simple adaptive controller," IEEE Transactions on Automatic Control, vol. 41, no. 9, pp. 1352-1358, 1996.
[22] M. R. Akella and K. Subbarao, "A novel parameter projection mechanism for smooth and stable adaptive control," Systems \& Control Letters, vol. 54, no. 1, pp. 43-51, 2005.

[23] R. T. H. Alden and A. A. Shaltout, "Analysis of damping and synchronizing torques-part 1: a general calculation method," IEEE Transactions on Power Apparatus and Systems, vol. 98, no. 5, pp. 1696-1700, 1979.

[24] E. Abu-Al-Feilat, M. Bettayeb, H. Al-Duwaish, M. Abido, and A. Mantawy, "A neural network-based approach for on-line dynamic stability assessment using synchronizing and damping torque coefficients," Electric Power Systems Research, vol. 39, no. 2, pp. 103-110, 1996.

[25] M. Ranlöf, J. Bladh, and U. Lundin, "Use of a finite element model for the determination of damping and synchronizing torques of hydroelectric generators," Electrical Power and Energy Systems, vol. 44, pp. 844-851, 2013. 


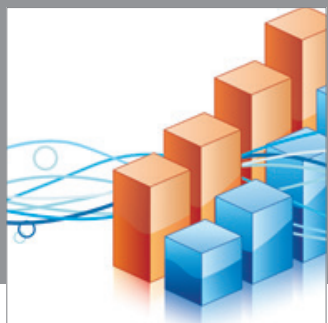

Advances in

Operations Research

mansans

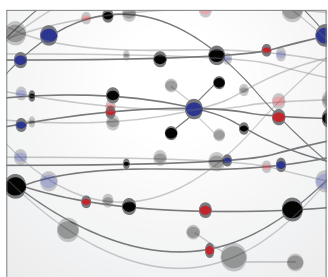

The Scientific World Journal
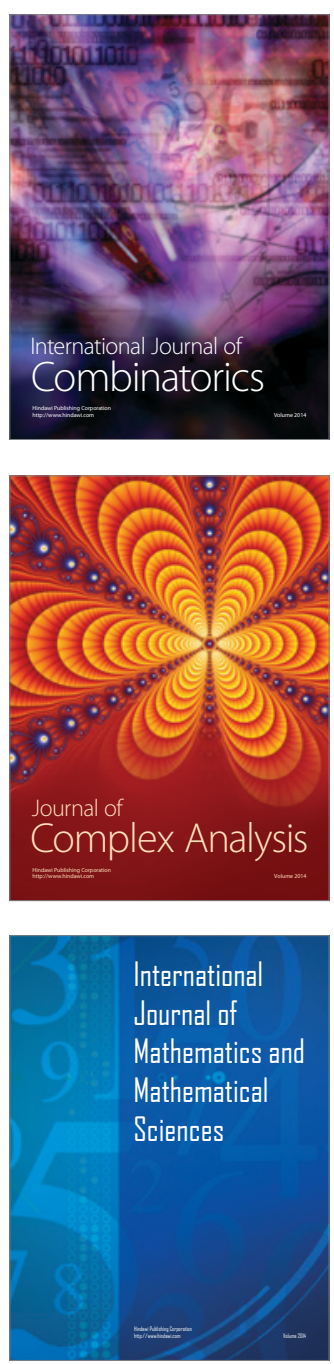
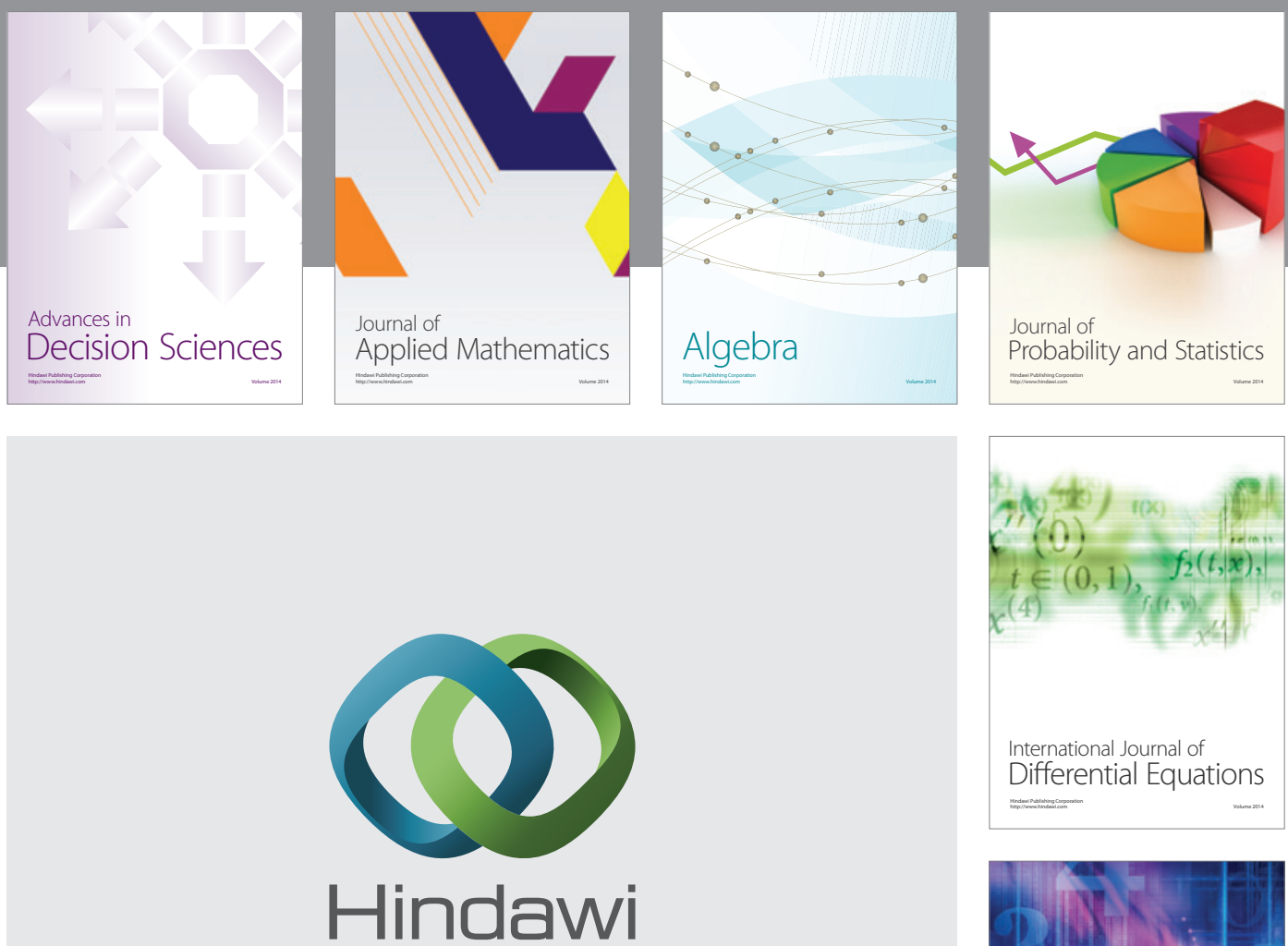

Submit your manuscripts at http://www.hindawi.com
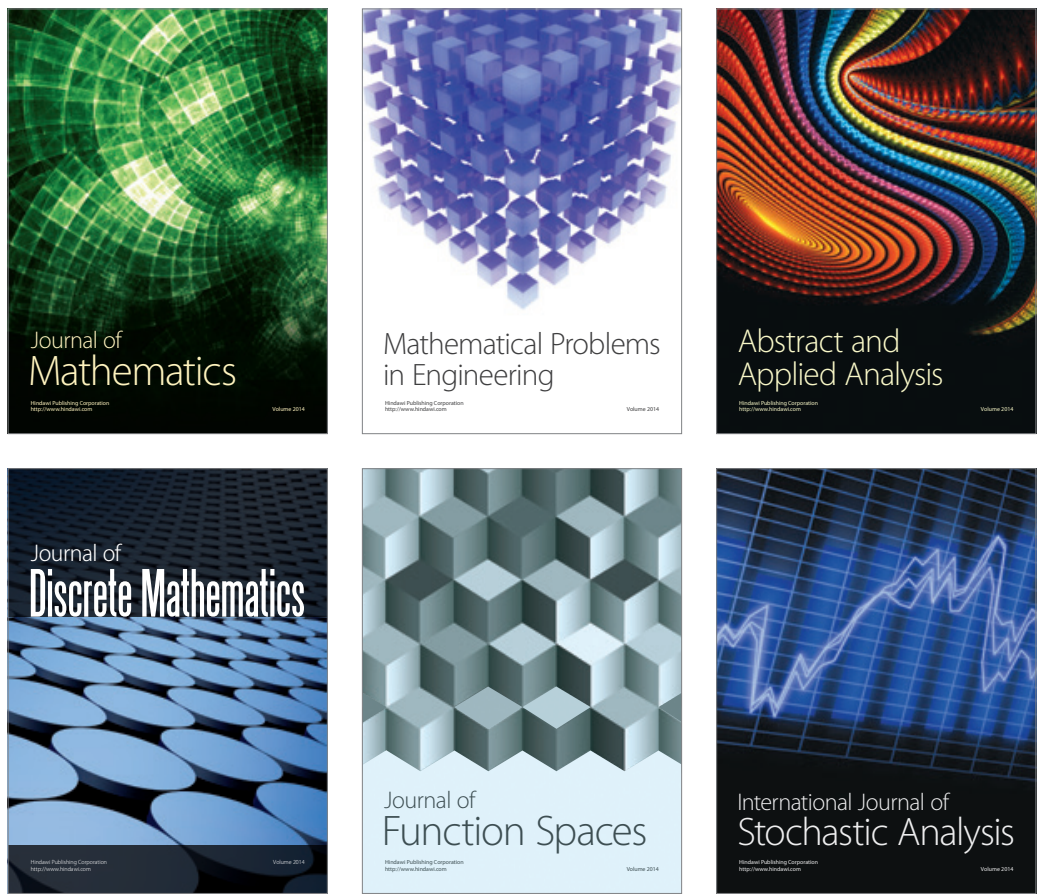

Journal of

Function Spaces

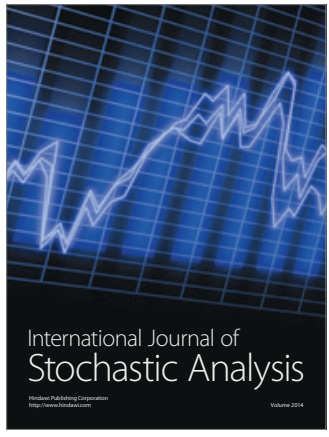

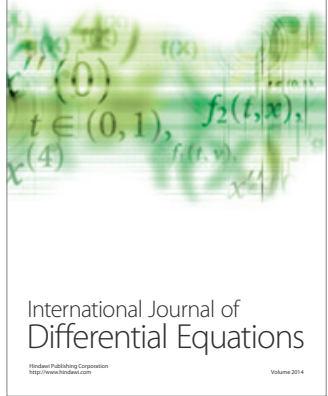
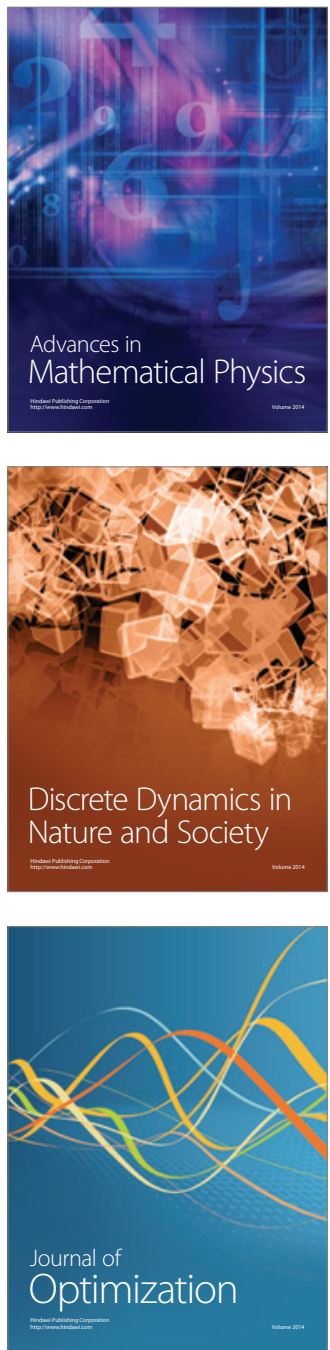